\title{
Exploring Limitations of User Interface Design to Understanding the Gap Between Technology and Seniors
}

\author{
Monika JINGAR ${ }^{\mathrm{a}, 1}$, Helena LINDGREN ${ }^{\mathrm{a}}$ and Madeleine BLUSI ${ }^{\mathrm{b}, \mathrm{c}}$ \\ ${ }^{a}$ Department of Computing Science, Umeå University, Sweden \\ ${ }^{\mathrm{b}}$ Department of Community Medicine and Rehabilitation, Umeå University, Sweden \\ ${ }^{\mathrm{c}}$ Department of Nursing, Umeå University, Sweden
}

\begin{abstract}
Participating in social activities promotes healthy ageing, whereas loneliness and isolation are known to cause adverse effects on both physical and mental wellbeing. Technology that exists in society today can facilitate healthy ageing. However, a gap can be seen between seniors and technology in today's internet and communication technological device's user interfaces. Due to limited prior knowledge of interacting with touch screen devices, seniors sometimes have difficulties using them. This research aims to explore the user interfaces and their elements designed using a human-centered design methodology by involving seniors as activate participants in the design process. This work's outcome can improve current user interface design practices in touch screen devices, which might be seen as contributing step to understand the gap between seniors and technology.
\end{abstract}

Keywords. Digital divide, human-centered design, co-creation, user interface design, social isolation, loneliness, wellbeing, healthy ageing, technology, seniors, older adults

\section{Introduction}

The rapid and sudden growth in information and communication technologies (ICT) shifted the current world towards a more technology-centred digital world. In terms of interaction design, these technologies should be designed so that regardless of age, cognitive and physical impairments, a person should be able to use and interact with them effortlessly [1]. While people age over 65 are the group in a society where the use of the internet is increasing the most, they are still among those threatened to be excluded from society's opportunities due to the digital divide [2]. Over the past decades, research demonstrated the importance of social relations for older people's wellbeing [3] and health [4]. Digital technology has provided new opportunities to offer social participation for seniors, including those who do not have previous experience using digital technology [5].

Although touch-screen devices are made for smooth user experience (UX), without prior knowledge of different ways of interacting with ICT devices, universal gestures and different meanings of universally accepted user interface (UI) elements such as icons,

${ }^{1}$ Corresponding Author, Monika Jingar, Department of Computing Science, Umeå University, 901 87, Umeå, Sweden; E-mail: monikaj@cs.umu.se 
it becomes hard for a user to interact with such technologies [6]. One reason for this can be their mental model set for the WYSIWYG (What You See Is What You Get) type of interaction design popularised during the early era of web technologies. WYSIWYG interfaces are visually explained contrary to current touch screen interfaces, which are gesture base and requires thorough knowledge of interaction gestures and ongoing $\mathrm{UI} / \mathrm{UX}$ trends to understand the interface and interact with the system. Further exploring is needed to make advances in our understanding of UI design for seniors.

The research presented in this paper aimed to explore seniors' interaction with the UI design of tablet touch screen devices. For this, we engaged seniors to co-create a digital platform for promoting social activity participation to prevent social isolation and loneliness by involving them as active participants using human-centered design (HCD) methodology. Efforts to understand older people's needs by involving them as active participants in the research process as co-creators is essential for designing user experiences aligned with seniors' expectations. During this research, particular emphasis was placed on understanding seniors' mental models to avoid ambiguity in UI design elements. The outcome from this research will guide the research community when designing UI for seniors with limited prior experience with digital technology.

\section{Methods}

A co-creation design process using HCD methodology was carried out by a multidisciplinary research team involving expert from healthcare, HCI and computer science. The co-creation process involves four major steps: 1) Understanding the use of context and users' requirements, 2) Ideation, 3) Prototyping and 4) Testing.

The target user group was seniors 70 years or older; inclusion criteria were individuals with independent living conditions and interest in developing social activities for promoting healthy and active ageing. Through advertisement at senior activity centres, 11 voluntary participants were recruited. Prior experience with digital technology was not a requirement. Ethical approval was granted by the Regional Ethical Review Board in Umeå, Sweden (Dnr 2017/50-31).

Understanding and ideation phase - To get insights from the target user group about their preferences, needs and expectations from a digital application to promote social participation, two focus groups were held. Two researchers mediated the focus group, and notes were taken during the focus group. A debriefing session was held after the focus group, and further notes were taken. All notes were analysed and translated into requirements and ideas, which was then used for the next step for HCD methodology, the ideation phase.

Prototyping phase - Requirements from the previous phase were carried on to the next step of prototyping. An iterative process was carried out to design a low-fidelity prototype. Shneiderman's eight golden rules of interface design [7] and design guidelines [8] were used to guide the designing of UI. These rules were selected since they focused more on UI design principles. Usability heuristics for UI design proposed by Nielsen [9] were used during the heuristic evaluation of the high-fidelity prototype. By doing this we involved two layers of evaluation of designed UI design guidelines and principles accepted by HCI community. The low-fidelity prototype was translated into a highfidelity interactive prototype using Axure RP software, aimed for tablet devices for the android platform, as shown in Figure:1. HCI researcher conducted the heuristic 
evaluation on the high-fidelity prototype to evaluate the proposed UI and find common errors in overall usability before testing with actual users.

Testing phase - The user test sessions were held by two researchers, where one carried the testing and one took the role of observer. Each test session followed a protocol with specified tasks to be performed by each participant using the think-aloud method. UX strategies were employed, which took the limitations such as a clash of expectations from an actual application versus a prototype into account. During the testing sessions, priority was given to understand the UI and its elements to gain insights for UI design improvements for seniors with limited prior experience with digital interfaces.

Data collection and analysis - During test sessions, the participant's voice and the screen were recorded to learn about their interaction patterns and hand gestures, as shown in Figure: 1 and through written notes. Semi-structured interviews were conducted immediately following the testing sessions, covering the following areas: 1) how it felt to use the application, 2) positive experiences, 3) difficulties, 4) disappointments and suggestions for improvement; to gain further insights about the overall UX after using the UI of the prototype. The video was analysed to investigate the participants' gestures and interaction patterns while interacting with the UI and tablet device. Notes and interviews were analysed using inductive content analysis.

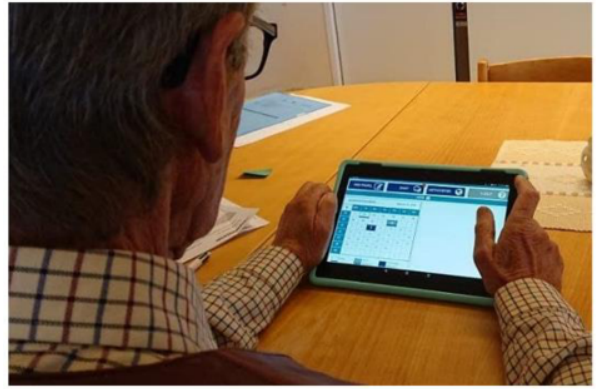

(a)

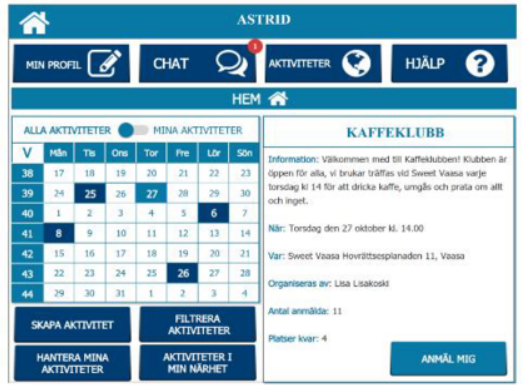

(b)

Figure 1. (a) Testing Setup with Participant and Table Interface; (b) User interface of high-fidelity prototype.

\section{Results}

None of the 11 participants had used a tablet before participating in this test. Three persons had used a PC; nine had a smartphone, from which eight of them used it only for telephone calls. Two persons had never used a smartphone but had button-type cellphones. Results were summarised into four main categories. Further, recommendations on how to modify current UI design practices for touch screen devices based on the results are included.

\subsection{Interaction and Usability Factors}

It was observed that the participants interact with the digital tablet's UI the same way they would interact with tangible items in the real-world such as a remote controller. During the testing session, the participants tend to click a clickable button for a longer time, which was interpreted as the hold action by the system resulting in no action and making the participants think that they have made a mistake. A possible solution could be to adapt and personalised ways of interaction according to the user's interaction style 
and patterns. Register the action even though it is prolonged and give cue in the form of tangible and visual feedback to the user before accepting their interaction by making changes in the UI.

\subsection{Ambiguity of Icons and Symbols}

First Participants find the icons and symbols used in the prototype to be more appealing than the text. The icons and UI elements used in the prototype were universally accepted and recognised for their dedicated meaning. However, participants find them confusing with their own interpretation of the same icons and symbols, creating ambiguity in the meaning behind them. For example, the symbol of hotel was used to indicate the main page globally called home page. The participants hesitate to click on that button as they thought it would initiate contact with an actual hotel. Another example includes a globally accepted green tick mark " $\checkmark$ " as a symbol of positive confirmation. However, participants associate this tick mark as the mark they received from their teachers to mark wrong entries in their childhood, making the meaning behind the symbol opposite to how we used it in the prototype. A possible solution could be to give users a tool to create and personalise a set of icons and symbols to design their own UI elements.

\subsection{Participants' Subjective Experience of the UI}

Participants were unanimously positive about the simplicity of the design. Even though none of the participants had used a tablet before and did not receive instructions "howto" during the test, they figured out the functions within the first couple of minutes. Through content analysis, a theme was formulated "co-created user interface was intuitive and inspiring" based on three categories: 1) easy to use, 2) easy to figure out, and 3) woke desire to learn. Quotes from participants are: "I was afraid I wouldn't be able to do it, but it was surprisingly easy", "I had never seen a tablet before, yet it feels familiar", and "I feel excited, I want to use it more, and wonder what else I can do with this, it has waken my curiosity".

\subsection{HCD Methodology and Seniors as Active Participants}

Using HCD methodology, we included seniors as active participants, as experts and as individuals and enabled them to comment, express and participate in the design process to enable inclusion of this group in this technological world. Seniors are often ignored as active participants in any design process, as noticed in the past [10]. Older adult's attitude for using digital devices is known to be heterogeneous. HCD methodology showed an added benefit for this specific research work in terms of getting more empathetic insights and views about seniors' actual potential to learn and be included in the world of technology.

\section{Discussion and Conclusion}

The research domain of seniors and ICT interaction has a rich literature. The role of gestures in the interaction paradigm has been explored in [6] using the familiarity-based design approach. The effectiveness of visual design focusing on colour, text, and layout 
of an interface was explored in [11]. Our research used HCD methodology and focus on interaction styles, design elements and findings components which creates a barrier for seniors during interaction with ICT. That helped us follow a human-centered process that starts with the user, emphatically creates design artifacts that address real user needs, and then tests those artifacts with real users. Seniors' attitude towards using digital devices is known to be heterogeneous. Thus, getting insights about specific needs, different preferences and views on using and interacting with the digital platforms with limited prior experience were essential to gather a more in-depth understanding of what is lacking in current UI design practices.

During the design process, seniors' thoughts, preferences and needs were collected, discussed and translated into requirements. That formed the base for a high-fidelity prototype, which was later tested, emphasising learning about the UI-related limitations and understanding various interaction styles and patterns. To conclude, digitally inexperienced seniors may have a predetermined understanding of standardised icons' meaning, thus misinterpreting icons' meaning. Hence standardised UI symbols can create obstacles for this target group to understanding how-to-use ICT devices. The results of this study indicate that digitally inexperienced seniors are willing to learn but need coaching to help them understand the meaning of standardised icons and symbols to promote digital inclusion. If left on their own, there is a risk they misinterpret unnecessary obstacles and hesitate continuous use of ICT devices. That is an essential finding, indicating that carefully co-created UI may promote seniors towards digital inclusion. The analysis also showcased significant issues relating to interaction and usability factors such as ambiguity of icons and symbols, which, when resolved, can enhance the overall user experience and promote using of technology among seniors.

\section{References}

[1] Chan MY, Haber S, Drew LM, Park DC. Training Older Adults to Use Tablet Computers: Does It Enhance Cognitive Function? Gerontologist. 2016 Jun;56(3):475-84.

[2] Delello JA, McWhorter RR. Reducing the digital divide: Connecting older adults to iPad technology. Journal of Applied Gerontology. 2017 Jan;36(1):3-28.

[3] Nicholson NR. A review of social isolation: an important but underassessed condition in seniors. J Prim Prev. 2012;33(2-3):137-152.

[4] Ferlander S. The importance of different forms of social capital for health. Acta Sociol. 2007;50:115-28.

[5] Blusi M, Kristiansen L, Jong M. Exploring the influence of Internet-based caregiver support on experiences of isolation for older spouse caregivers in rural areas: A qualitative interview study. Int $\mathrm{J}$ Older People Nurs. 2015;10(3):211-220.

[6] Leonardi C, Albertini A, Pianesi F, Zancanaro M. An exploratory study of a touch-based gestural interface for elderly. InProceedings of the 6th nordic conference on human-computer interaction: Extending boundaries 2010 Oct 16 (pp. 845-850).

[7] Lidwell W, Holden K, Butler J. Universal principles of design, revised and updated: 125 ways to enhance usability, influence perception, increase appeal, make better design decisions, and teach through design. Rockport Pub; 2010.

[8] Zaphiris P, Ghiawadwala M, Mughal S. Age-centered research-based web design guidelines. InCHI'05 extended abstracts on Human factors in computing systems 2005 Apr 2 (pp. 1897-1900).

[9] Nielsen J. Enhancing the explanatory power of usability heuristics. InProceedings of the SIGCHI conference on Human Factors in Computing Systems 1994 Apr 24 (pp. 152-158).

[10] Blusi M, Asplund K, Jong M. Older family carers in rural areas: experiences from using caregiver support services based on Information and Communication Technology (ICT). Eur J Ageing. 2013;10(3):191-9.

[11] Zongliang B, Guang F, Ping W. Elderly-Oriented Design of User Interface of Agedness Internet Products Based on Synesthesia Thinking. InProceedings of the 2020 The 2nd World Symposium on Software Engineering 2020 Sep 25 (pp. 51-54). 